OPEN ACCESS

Edited by:

Błażej Misiak,

Wroclaw Medical University, Poland

Reviewed by:

Chuanjun Zhuo,

Tianjin Medical University General

Hospital, China

Daniel Berge,

Institut Hospital del Mar

d'Investigacions Mèdiques (IMIM),

Spain

*Correspondence: Jungsun Lee

js_lee@amc.seoul.kr

Specialty section: This article was submitted to

Schizophrenia,

a section of the journal

Frontiers in Psychiatry

Received: 10 March 2018 Accepted: 05 June 2018

Published: 26 June 2018

Citation:

Shon S-H, Yoon W, Kim H, Joo SW, Kim Y and Lee J (2018) Deterioration in Global Organization of Structural Brain Networks in Schizophrenia: A Diffusion MRI Tractography Study. Front. Psychiatry 9:272.

doi: 10.3389/fpsyt.2018.00272

\section{Deterioration in Global Organization of Structural Brain Networks in Schizophrenia: A Diffusion MRI Tractography Study}

\author{
Seung-Hyun Shon ${ }^{1}$, Woon Yoon ${ }^{1}$, Harin Kim ${ }^{2}$, Sung Woo Joo ${ }^{3}$, Yangsik Kim ${ }^{4}$ and \\ Jungsun Lee ${ }^{1 *}$
}

${ }^{1}$ Department of Psychiatry, Asan Medical Center, University of Ulsan College of Medicine, Seoul, South Korea, ${ }^{2}$ Korea Armed Forces Capital Hospital, Department of Psychiatry, Seongnam, South Korea, ${ }^{3}$ Republic of Korea Marine Corps, Pohang, South Korea, ${ }^{4}$ Graduated School of Medical Science and Engineering, Korea Advanced Institute for Science and Technology, Daejeon, South Korea

Schizophrenia is a heterogenous neuropsychiatric disorder with varying degrees of altered connectivity in a wide range of brain areas. Network analysis using graph theory allows researchers to integrate and quantify relationships between widespread changes in a network system. This study examined the organization of brain structural networks by applying diffusion MRI, probabilistic tractography, and network analysis to 48 schizophrenia patients and 24 healthy controls. T1-weighted MR images obtained from all participants were parcellated into 87 regions of interests (ROls) according to a prior anatomical template and registered to diffusion-weighted images (DWI) of the same subjects. Probabilistic tractography was performed to obtain sets of white matter tracts between any two ROls and determine the connection probabilities between them. Connectivity matrices were constructed using these estimated connectivity probabilities, and several network properties related to network effectiveness were calculated. Global efficiency, local efficiency, clustering coefficient, and mean connectivity strength were significantly lower in schizophrenia patients $(p=0.042, p=0.011, p=0.013, p$ $=0.046)$. Mean betweenness centrality was significantly higher in schizophrenia ( $p$ $=0.041)$. Comparisons of node wise properties showed trends toward differences in several brain regions. Nodal local efficiency was consistently lower in the basal ganglia, frontal, temporal, cingulate, diencephalon, and precuneus regions in the schizophrenia group. Inter-group differences in nodal degree and nodal betweenness centrality varied by region and showed inconsistent results. Robustness was not significantly different between the study groups. Significant positive correlations were found between t-score of color trails test part- 1 and local efficiency and mean connectivity strength in the patient group. The findings of this study suggest that schizophrenia results in deterioration of the global network organization of the brain and reduced ability for information processing.

Keywords: schizophrenia, diffusion MRI, probabilistic tractography, network analysis, connectivity 


\section{INTRODUCTION}

Schizophrenia is a debilitating mental disorder with an onset in early adulthood, a chronic course, and a considerable disease burden $(1,2)$. Common clinical domains include delusion, hallucination, disorganized thought, and decline of cognitive abilities, with the manifestation and severity of symptoms varying from patient to patient (3). This symptom heterogeneity makes it difficult to understand the pathophysiology of the disease (4). From this background, it is believed that schizophrenia is a disease associated with varying degrees of impairment in a wide range of brain areas, rather than distinct changes in focal brain lesions (5-7). Therefore, for a more objective diagnosis and understanding, a biomarker that can comprehensively analyze schizophrenia-related changes in the whole brain is considered to be important.

Various methods have been used to detect minute changes in brain structure and to analyze them in an integrated manner. Diffusion-weighted imaging (DWI) is a noninvasive method that displays parameters related to the diffusion of water molecules and can be used to provide diffusion tensor image (DTI) by measuring the diffusion of water in many different directions. The diffusion of water in the brain is restricted by cell membranes and cellular structures, and these limitations cause anisotropic diffusion or directional preference in diffusion. Thus, changes in diffusivity measured by DTI can reflect neural tissue damage or the organization of neural fibers. Rather than considering only the single dominant tensor orientation for each voxel, probabilistic tractography (8) repeatedly samples the data and generates many streamline tracts connecting two brain locations, with the density of these tracts reflecting the probability of the voxel-level diffusion directions. However, previous research has revealed that conventional tensor models are subject to several problems with regard to the accurate tracking of complex fiber structures, such as crossing and fanning fibers $(9,10)$. Tractography methods have therefore been extended to model up to two major diffusion directions in each voxel, with one or two voxel-level directions then being selected for fiber tracking (11). These tractography methods using two-fiber modeling have a higher sensitivity for the detection of multiple fiber populations in the whole brain (11). After generating a set of streamlines for the whole brain, the likelihood of connection between the any two regions can be estimated. By applying tractography, it is possible to reconstruct a brain network that considers the specific regions of the brain as nodes, and the connections between the regions as edges.

Network analysis is a branch of mathematics that describes a system as a graph, which is a set of nodes connected by a set of edges, and then analyses the topological characteristics of the graph (12). The degree of brain structure abnormality between subjects may be quantitatively compared by comparing group differences in metric values obtained from such networks. Network analysis allows researchers to integrate and quantify the relationships in the multivariate data representing the

Abbreviations: ROIs, Regions of interests; DWI, diffusion-weighted images; DTI, diffusion tensor image; MCMC, Markov chain Monte Carlo. neural network system. Previous studies analyzing reconstructed networks have revealed connectivity disturbances in brain structure in schizophrenia subjects, on the basis of abnormalities in several topological network properties. In short, the main findings concerning the anatomical brain networks of schizophrenia patients were less optimal organization, a less efficiently connected network, less connectivity strength, and reduced hierarchy (13). Researchers have interpreted these results as being suggestive of a decrease in information integration ability and an abnormality in neural development (13-15). Moreover, these findings of altered network organization have led to the hypothesis that structural abnormalities in the brain network could act as biomarkers for inherited genetic vulnerability, and that the more deteriorated the network, the more susceptible it may be to progressive white matter damage, which in turn may bring about more significant functional decline $(16,17)$.

Robustness is one of the network properties that may be analyzed; it is a concept developed through attempts to understand the brain's stability to physical damage. Robustness simulation, which involves quantifying changes in network properties after removal of components of the brain network, has revealed that the brain is more stable to random damage, but is more vulnerable to target deletion, which is the removal of specific regions in a particular order (18). To our knowledge, less network studies have investigated the robustness of the brains of schizophrenia patients than have examined other network properties, and the results have been inconsistent. Although several previous studies reported that schizophrenia groups showed relatively low resilience to damage compared to healthy control groups $(19,20)$, there is also one report that found that a healthy control group was affected more severely by the target deletion of nodes (21).

From the viewpoint of the distribution of connectivity, the normal brain network has a topological characteristic in which a relatively small number of regions (i.e., nodes constituting the network) are involved with the majority of connections (22). The "brain hub" (23) is a term that has been used to refer to these specific brain areas, which are deeply involved in the integration of information and have a high degree of centrality. In normal brain network organization, the hub area shows a high degree, a low cluster coefficient, and a high degree of centrality, and is suggested to be an important area contributing to the integration of information and the stability of the network $(4,24)$. Previous findings have suggested disruption to hub regions, with research strongly implicating a less central position of the prefrontal hubs, and moderately implicating abnormalities of the limbic, temporal, and parietal hubs (4). As the number of hubs in the brain network is relatively small and the importance of each node to the network varies, it is necessary to not only analyze the characteristics of the entire network, but to also compare the characteristics of each of the regional network properties when making comparisons between healthy controls and patients with schizophrenia.

The study of brain networks with diffusion MRI can facilitate the evaluation of the topologic characteristics of the entire brain structure, although it is difficult to accurately reflect the 
organization of complex brain fibers. Although several network studies have been conducted to date, definitive findings have not yet been achieved. Therefore, to expand the understanding of the structural characteristics of the brain in schizophrenia patients, this study aimed to assess the global network properties of the brain using extended multi-fiber probabilistic tractography. We reconstructed the brain network of each subject using diffusion imaging probabilistic tractography, and then we compared group differences in global network properties. In addition, we compared differences in nodal network properties and conducted a robustness simulation to better understand the stability and regional organization of the brain networks.

\section{MATERIALS AND METHODS}

\section{Participants}

Subjects were recruited from the Asan Medical Center in Seoul, Korea. Forty-eight schizophrenia patients were enrolled, with all patients meeting the Diagnostic and Statistical Manual of Mental Disorders-IV-Text Revision (DSM-IV-TR) criteria. They were all right-handed, between the ages of 20 and 40 years old, and had no other known diseases that could affect brain function. They had all displayed psychotic symptoms such as delusions or hallucinations for $<5$ years. Twenty-four healthy controls who did not have any Axis I psychiatric diagnosis were enrolled. Furthermore, the healthy controls did not have any first-degree relatives with an Axis I psychiatric diagnosis. In addition, subjects were excluded if they were unable to complete MRI scanning sessions.

Written informed consent was obtained from all subjects. Ethnical approval for the study was obtained from the local Institutional Review Board, Asan Medical Center, Seoul, Korea.

\section{Neuroimaging Acquisition}

MRI was performed on a 3-Tesla scanner with an eight channel SENSE head coil (Philips Achieva). Structural T1-weighted images were acquired with a turbo field echo sequence (FOV: 240 $\times 240 \times 170 \mathrm{~mm}$, voxel size: $1 \times 1 \times 1 \mathrm{~mm}$, TE/TR: 4.6/4.9 ms). DWI images were acquired with an echo planar imaging (EPI) diffusion-weighted sequence. One baseline ( $b$ factor $=0 \mathrm{~s} / \mathrm{mm}^{2}$ ) image and 32 diffusion-weighted gradient directions (b factor $=1,000 \mathrm{~s} / \mathrm{mm}^{2}$ ) were acquired (FOV: $224 \times 224 \times 135 \mathrm{~mm}$, voxel size: $2 \times 2 \times 3 \mathrm{~mm}$, TE/TR: $70 / 5,422 \mathrm{~ms}$, flip angle: $\left.90^{\circ}\right)$. Inappropriate images were found via visual inspection and excluded from further analyses.

Anisotropic voxel affects the distribution of anisotropic signal to noise ratios and can cause directional errors in the fiber tracking algorithm (25). Therefore, DTI images were up-sampled in this study to convert anisotropic voxel size $2 \times 2 \times 3 \mathrm{~mm}$ to isotropic voxel size $2 \mathrm{~mm}$ using Slicer V.4.4 (26). Corrections for motion and eddy current artifacts were performed via affine whole brain registration to the b0 baseline using FLIRT in FSL (v 6.0, FMRIB Software, Oxford, UK). During the correction process, gradients or subjects were not discarded by applying a specific threshold. In this study, we did not perform field mapping correction (FDC) such as top up. Since the results of a previous study showed that this approach, which aligns diffusion images to anatomical image as the registration-template without using any additional data such as field-map, provides accurate correction similar to that of the FDC method. (27). The directions of the gradients were compensated for rotations during the process of correction for distortion using affine registration.

T1-weighted images were processed using the DesikanKilliany atlas of FreeSurfer V 5.3 to parcellate discrete anatomical regions of interest (ROIs) $(28,29)$. White matter and gray matter ROIs were combined into one ROI for each anatomical structure, resulting in 87 ROIs. This was followed by registration of the parcellated T1-weighted images to the DWI using FLIRT with six degrees of freedom (30).

\section{Network Reconstruction}

To track white matter streamlines, we applied a probabilistic tractography method using the Diffusion Toolbox in the FMRIB software library (FSL) $(8,11,31)$. Briefly, the probabilistic approach estimates the distribution of the diffusion parameters at each voxel using Metropolis-Hastings Markov chain Monte Carlo (MCMC) sampling (BEDPOST). Then, probabilistic tracking was performed by repeatedly sampling from the distributions. Five thousand samples were performed for each seed ROI and a set of streamlines passing through the given ROI were generated (probtrackx). To reflect the degree of connectivity and the influence of region size, the number of streamline tracts from one seeding ROI that passed through a given second ROI was divided by the total number of generated streamlines (i.e., the way-total value in FSL), to give a connectivity probability for the connection to the second ROI. The non-directional connectivity probability between two ROIs was defined by averaging the two probabilities attained from tracking from each given ROI.

Brain networks were then reconstructed from the collection of ROIs and calculated connectivity probabilities, resulting in an association matrix. Each network was represented as a graph, $\mathrm{G}=(\mathrm{V}, \mathrm{E})$, consisting of a set of nodes $\mathrm{V}$ (representing 87 ROIs) and connections $\mathrm{E}$ between the nodes (representing connectivity probability between nodes). To remove weak or spurious connections, we applied a threshold to the connectivity probabilities, with the lowest $10 \%$ of connections in each subject's network being discarded. Detailed information on the 87 ROIs used in the study is given in Supplementary Table 1.

\section{Network Examination}

Several network properties of the reconstructed brain networks were evaluated to characterize their organization. These network properties were all based on the non-directional weighted matrices and were calculated using the MATLAB-based Brain Connectivity Toolbox (http://www.brain-connectivity-toolbox. net) (32). As the importance of the topological features of each brain region may differ between regions, the nodal network properties were also analyzed.

The nodal local efficiency, degree, and betweenness centrality were calculated to describe the connectivity of the specific brain regions. Nodal local efficiency describes the inverse values of the shortest path length between direct neighbors of a given node, degree represents the number of all connections to a given node, 
and betweenness centrality is related to the number of shortest paths in a network passing through a given node. Nodal local efficiency quantifies the segregation properties of the network (33), while degree and betweenness centrality allow assessment of the importance of each node $(32,34)$.

Global properties including the mean connectivity strength, global efficiency, clustering coefficient, local efficiency, and mean betweenness centrality were calculated to define the topological characteristics of the whole brain network. Mean connectivity strength is a global measure of the average connectivity probability values of all connections, and is related to the strength of the entire network (35). Global efficiency is the average of the inverse values of the shortest path length between all pairs of nodes in the network, and quantifies global network integration (33). The clustering coefficient is the global likelihood that the direct neighbors of a given node are interconnected, and quantifies network segregation (36). Local efficiency and mean betweenness centrality were calculated as the average of the node-wise property values.

\section{Robustness Simulation}

Robustness is an indicator of network stability when brain damage is present (18). To evaluate the stability of the network, iterative elimination of nodes was simulated. Of the 87 ROIs, 40 nodes were removed one by one in order of decreasing nodal degrees in the brain network of each patient. During node deletion, the global efficiency and global clustering coefficient of each subject's brain network were calculated repeatedly, to track the topological features of the damaged network, after which, group means of changes in network properties according to the number of deleted nodes and differences in group means of differences between patients and control groups were examined. Only 40 ROIs were removed from the network, because the removal of a larger number of ROIs resulted in errors when calculating the clustering coefficient.

\section{Clinical Assessments}

Symptom severity was assessed using the Korean version of Positive and Negative Syndrome Scale (PANSS) score (37, 38). To assess cognitive functioning, raw FSIQ scores were estimated using the Korean version of the Wechsler Adult Intelligence Scale (WAIS) and adjusted for age and gender (39). Additionally, color trails test parts 1, 2 (CTT-1,2) were used and the results were presented as T-scores adjusted for age and gender. CTT is an analog of the trails making test (TMT) but is less affected by cultural differences than TMT (40). While CTT part-1 evaluates visuomotor processing speed, CTT part-2 examines attention, visuomotor speed, delayed recall of declarative memory, working memory, and executive functioning (41). Overall psychosocial functioning was assessed using Global Assessment of Functioning (GAF) scale scores (42, 43).

\section{Group Comparison}

Demographic data were compared using independent $t$-tests or $\chi^{2}$-tests for categorical variables. Differences in network properties between study groups were compared using a twotailed Student's $t$-test or a Mann Whitney U-test. Multiple comparison corrections were applied to the comparisons of nodal properties by controlling the false discovery rate at 5\% (44). A linear mixed model with the number of removed nodes and diagnosis as a fixed effect and subject as a random effect were used to characterize the resilience of the network properties during robustness simulation.

\section{RESULTS}

\section{Demographic and Clinical Characteristics}

There were no significant differences in mean age or gender between the groups. The mean IQ scores and CTT- 2 t scores were lower in patient group than in the healthy control group $(p<$ 0.001). Table 1 lists the demographic and clinical characteristics of the participants by group.

\section{Global Network Properties}

Patients with schizophrenia showed lower global efficiency, reduced local efficiency, reduced clustering coefficient, and reduced mean connectivity strength in comparison with the healthy control group. Mean betweenness centrality $(245.1399 \pm$ 10.2767 vs. $239.6925 \pm 10.7912 ; F=0.013 ; p=0.041$ ) was higher in schizophrenia patients than in controls. Table 2 lists the group differences in global network properties.

\section{Nodal Network Properties}

None of the findings reached the FDR-threshold. The patient group showed lower nodal local efficiency at an uncorrected $(p$ $<0.05$ ) level in several areas, including the basal ganglia (left caudate nucleus and left nucleus accumbens), frontal lobe (left pars orbitalis, right caudal middle frontal gyrus, right medial orbitofrontal cortex, right pars opercularis, right precentral gyrus, and right superior frontal gyrus), temporal lobe (bilateral hippocampus, left superior temporal gyrus, left temporal pole, right inferior temporal gyrus, right superior temporal gyrus, and right transverse temporal gyrus), cingulate cortex (left caudal anterior cingulate cortex, left rostral anterior cingulate cortex, and right rostral anterior cingulate cortex), diencephalon

TABLE 1 | Demographic information on the schizophrenia (SPR) patients and healthy control subjects.

\begin{tabular}{lcccc}
\hline & SPR & Healthy control & $\boldsymbol{F}$ or $\boldsymbol{\chi}^{\mathbf{2}}$ & $\boldsymbol{p}$ \\
\hline Age, years, mean $(S D)$ & $28.9(6.2)$ & $30.0(5.3)$ & 1.66 & 0.418 \\
Gender, male, $n(\%)$ & $19(39.6)$ & $9(37.5)$ & 0.29 & 0.864 \\
FSIQ, mean (SD) & $97.8(15.5)$ & $120.1(9.2)$ & 7.86 & $<0.001$ \\
CTT-t 1, mean (SD) & $48.6(14.4)$ & $54.5(7.5)$ & 1.88 & 0.064 \\
CTT-t 2, mean (SD) & $47.0(13.7)$ & $63.8(20.5)$ & 4.15 & $<0.001$ \\
GAF, mean (SD) & $39.8(19.3)$ & - & - & - \\
PANSS, mean (SD) & $61.0(14.7)$ & - & - & - \\
\hline
\end{tabular}

$F S I Q$, Full scale intelligence quotient; CTT-t, $t$-score for Color trails test; GAF, Global assessment of functioning; PANSS, Positive and negative syndrome scale. 
TABLE 2 | Global network properties of schizophrenia (SPR) patients and healthy control subjects.

\begin{tabular}{|c|c|c|c|c|}
\hline \multirow{2}{*}{$\begin{array}{l}\text { Network } \\
\text { properties }\end{array}$} & \multirow{2}{*}{$\begin{array}{c}\text { SPR } \\
\text { Mean (SD) }\end{array}$} & \multirow{2}{*}{$\begin{array}{l}\text { Healthy control } \\
\text { Mean (SD) }\end{array}$} & \multirow[t]{2}{*}{$\boldsymbol{F}$} & \multirow[t]{2}{*}{$p$} \\
\hline & & & & \\
\hline $\begin{array}{l}\text { Global } \\
\text { efficiency }\end{array}$ & 1.14E-1 (2.26E-3) & 1.15E-1 (2.65E-3) & 0.47 & 0.042 \\
\hline $\begin{array}{l}\text { Local } \\
\text { efficiency }\end{array}$ & 1.02E-2 (7.87E-4) & 1.08E-2 (1.13E-3) & 3.22 & 0.011 \\
\hline $\begin{array}{l}\text { Clustering } \\
\text { coefficient }\end{array}$ & 7.64E-3 (6.42E-4) & 8.12E-3 (9.25E-4) & 3.20 & 0.013 \\
\hline $\begin{array}{l}\text { Mean } \\
\text { betweenness } \\
\text { centrality }\end{array}$ & 245.14 (10.28) & 239.69 (10.80) & 0.01 & 0.041 \\
\hline $\begin{array}{l}\text { Mean } \\
\text { connectivity } \\
\text { strength }\end{array}$ & $3.48 \mathrm{E}-1$ (1.28E-3) & 3.55E-1 (1.63E-3) & 1.97 & 0.046 \\
\hline
\end{tabular}

(left thalamus and left ventral diencephalon), and parietal (left precuneus) area.

The schizophrenia group showed increased nodal degree values in the left pars orbitalis, right lateral orbitofrontal cortex, right hippocampus, and right ventral diencephalon regions, while the healthy control group showed increased values in the right transverse temporal gyrus, right supramarginal gyrus, and right nucleus accumbens regions. The nodal betweenness centrality values in the right entorhinal cortex area of the schizophrenia patients were higher than in the healthy control group.

Table 3 lists the ROIs showing differences at uncorrected $p$ values of $<0.05$ for each of the nodal network properties.

\section{Robustness of Brain Structural Networks}

Scatter plots of the number of deleted nodes and changes in network properties illustrate the resilience of the structural brain networks in the two study groups (Figure 1). Global efficiency decreased continuously as the number of removed nodes increased. However, global efficiency did not show a significant group-by-number of deleted node interaction $(p=$ 0.184). Plotting of the clustering coefficient resulted in a $\mathrm{U}$ shaped pattern in which the value of the property decreased in the early stage of the simulation, but then increased as the simulation progressed. In the case of the clustering coefficient, robustness could not be compared by linear mixed analysis because groupspecific variation of the property did not show linearity.

\section{Relationship Between Network Characteristics and Clinical Assessments}

In the patient group, there were significant positive correlations between CTT-1 t-score, and local efficiency and mean connectivity strength. The negative correlation of CTT-1 t-score with mean betweenness centrality was significant. CTT-2 t-score had a significant positive association with mean connectivity strength. In the patient group, there were no significant associations between IQ, GAF, and total PANSS score and network properties. In the control group, IQ raw score was significantly positively related to local efficiency, clustering coefficient, and mean connectivity strength.

Table 4 lists the relationship between network characteristics and clinical assessments for each subject group.

\section{DISCUSSION}

The schizophrenia group showed significantly lower values than the control group in global efficiency, local efficiency, clustering coefficient, and mean connectivity strength. Global efficiency is an indicator of network integration, and reduced values could imply that the ability for functional integration across the overall network is degraded. The clustering coefficient and local efficiency value were both lower in the schizophrenia group, which means that the degree of segregation across the overall network, i.e., the local connectedness, was lower. In addition, decreased overall connectivity strength was also reported, suggesting that overall connectivity between regions in the schizophrenia group was different to that in the control group. In a previous study of brain anatomical networks in drug naïve schizophrenia patients, Zhang et al. suggested that decreased connectivity strength in subnetworks affects the deterioration of global topological characteristics (19), which is in accord with our findings.

As suggested in a previous report (24), decreased global network parameters may result from either reduced interregional connectivity between brain regions, or disconnections in longer pathways. Our results are mostly consistent with previous studies, although different thresholds and anatomical templates have been applied $(45,46)$. However, when comparing properties related to network segregation (such as the clustering coefficient) with previous studies, between-study differences can be noted in the schizophrenia patients. (15, 45-47). Differences in tractography, thresholding, imaging sequence, and edge weight may have influenced these between-study differences related to network segregation. Exceptionally, the mean betweenness centrality of this study was significantly higher in the schizophrenia patients than the control subjects. This is inconsistent with previous studies, which showed that schizophrenia may be associated with abnormal network hub organization $(4,48)$. It has been reported that changes in centrality in schizophrenia are inconsistent between brain regions $(24,45)$. Thus, the effect on the mean betweenness centrality of schizophrenia might vary from region to region of the brain, and comparisons of the nodal measures should be considered for appropriate comparisons.

In comparison with the normal control subjects, the schizophrenia patients showed significantly lower nodal local efficiency values (at uncorrected $p<0.05$ levels) in the basal ganglia, frontal, temporal, cingulate, diencephalon, and precuneus regions. Previous studies have implicated these regions in the pathophysiology and symptom manifestation of schizophrenia, with the basal ganglia showing altered integration in schizophrenia, and striatal functional connectivity having been reported as a potential biomarker for predicting response to antipsychotics (49-51). Frontal and temporal areas are reported 
TABLE 3 | Regions showing differences in nodal network properties between subject groups (uncorrected level of $p<0.05$ ).

\begin{tabular}{|c|c|c|c|c|c|c|}
\hline \multirow[t]{2}{*}{ Area } & \multirow[t]{2}{*}{ ROI name } & \multicolumn{2}{|c|}{ Schizophrenia } & \multicolumn{2}{|c|}{ Health controls } & \multirow[t]{2}{*}{ Uncorrected $p$} \\
\hline & & mean & $S D$ & mean & $S D$ & \\
\hline \multicolumn{7}{|c|}{ NODAL LOCAL EFFICIENCY } \\
\hline \multirow[t]{6}{*}{ Frontal } & Left pars orbitalis & 4.26E-03 & $7.85 \mathrm{E}-04$ & 4.90E-03 & 1.11E-03 & $0.012^{\star}$ \\
\hline & Right caudal middle frontal gyrus & 1.15E-02 & 1.60E-03 & $1.24 \mathrm{E}-02$ & $1.78 \mathrm{E}-03$ & $0.049^{\star}$ \\
\hline & Right medial orbitofrontal cortex & $1.10 \mathrm{E}-02$ & 1.57E-03 & $1.23 \mathrm{E}-02$ & $1.76 \mathrm{E}-03$ & $0.006^{*}$ \\
\hline & Right pars opercularis & 8.87E-03 & 1.42E-03 & 9.86E-03 & $1.68 \mathrm{E}-03$ & $0.007^{\star}$ \\
\hline & Right precentral gyrus & 1.67E-02 & 2.61E-03 & 1.80E-02 & $2.76 \mathrm{E}-03$ & $0.032^{*}$ \\
\hline & Right superior frontal gyrus & 2.12E-02 & 2.27E-03 & 2.25E-02 & $2.56 \mathrm{E}-03$ & $0.022^{\star}$ \\
\hline \multirow[t]{7}{*}{ Temporal } & Left hippocampus & 9.04E-03 & 1.66E-03 & $1.00 \mathrm{E}-02$ & 2.07E-03 & $0.220^{*}$ \\
\hline & Left superior temporal gyrus & $1.24 \mathrm{E}-02$ & $1.44 \mathrm{E}-03$ & $1.31 \mathrm{E}-02$ & $1.35 \mathrm{E}-03$ & $0.039^{\star}$ \\
\hline & Left temporal pole & 6.95E-03 & 1.69E-03 & 7.99E-03 & $2.25 \mathrm{E}-03$ & $0.045^{\star}$ \\
\hline & Right hippocampus & 9.95E-03 & $1.55 \mathrm{E}-03$ & 1.10E-02 & $1.72 \mathrm{E}-03$ & $0.018^{\star}$ \\
\hline & Right inferior temporal gyrus & 7.82E-03 & $1.12 \mathrm{E}-03$ & 7.99E-03 & $1.23 \mathrm{E}-03$ & $0.017^{\star}$ \\
\hline & right superior temporal gyrus & 1.16E-02 & 1.53E-03 & $1.27 \mathrm{E}-02$ & $1.50 \mathrm{E}-03$ & $0.005^{\star}$ \\
\hline & Right transverse temporal gyrus & 6.96E-03 & 1.03E-03 & 7.60E-03 & $1.22 \mathrm{E}-03$ & $0.018^{\star}$ \\
\hline Parietal & Left precuneus & 9.74E-03 & $1.65 \mathrm{E}-03$ & 1.04E-02 & $1.25 \mathrm{E}-03$ & $0.024^{\star}$ \\
\hline \multirow[t]{3}{*}{ Cingulate } & Left caudal anterior cingulate cortex & 1.19E-02 & 1.94E-03 & 1.30E-02 & 2.06E-03 & $0.013^{\star}$ \\
\hline & Left rostral anterior cingulate cortex & $1.22 \mathrm{E}-02$ & 1.86E-03 & 1.33E-02 & $2.10 \mathrm{E}-03$ & $0.019^{\star}$ \\
\hline & Right rostral anterior cingulate cortex & 1.25E-02 & $1.88 \mathrm{E}-03$ & $1.40 \mathrm{E}-02$ & 2.33E-03 & $0.008^{\star}$ \\
\hline \multirow[t]{2}{*}{ Basal ganglia } & Left caudate nucleus & 1.27E-02 & $1.84 \mathrm{E}-03$ & $1.41 \mathrm{E}-02$ & $2.46 \mathrm{E}-03$ & $0.033^{*}$ \\
\hline & Left nucleus accumbens & 8.37E-03 & $1.55 \mathrm{E}-03$ & 9.44E-03 & $1.82 \mathrm{E}-03$ & $0.025^{\star}$ \\
\hline \multirow[t]{2}{*}{ Diencephalon } & Left thalamus & $1.18 \mathrm{E}-02$ & $1.76 \mathrm{E}-03$ & $1.26 \mathrm{E}-02$ & $1.84 \mathrm{E}-03$ & $0.049^{\star}$ \\
\hline & Left ventral diencephalon & 1.05E-02 & $1.02 \mathrm{E}-03$ & 1.12E-02 & 1.47E-03 & $0.045^{\star}$ \\
\hline \multicolumn{7}{|c|}{ NODAL DEGREE } \\
\hline \multirow[t]{2}{*}{ Frontal } & Left pars orbitalis & 61.10 & 4.35 & 58.67 & 5.74 & $0.048^{\star \star}$ \\
\hline & Right lateral orbitofrontal cortex & 78.40 & 3.22 & 76.58 & 2.75 & $0.007^{\star \star}$ \\
\hline \multirow[t]{2}{*}{ Temporal } & Right hippocampus & 83.06 & 2.45 & 82.79 & 1.06 & $0.030^{\star \star}$ \\
\hline & Right transverse temporal gyrus & 77.60 & 3.47 & 80.04 & 2.16 & $0.003^{\star}$ \\
\hline Parietal & Right supramarginal gyrus & 80.85 & 2.32 & 82.00 & 1.67 & $0.050^{\star}$ \\
\hline Basal ganglia & Right nucleus accumbens & 76.98 & 4.14 & 79.13 & 2.88 & $0.033^{\star}$ \\
\hline Diencephalon & Right ventral diencephalon & 84.54 & 1.03 & 83.71 & 1.60 & $0.027^{\star \star}$ \\
\hline \multicolumn{7}{|c|}{ BETWEENNESS CENTRALITY } \\
\hline Parietal & Right entorhinal cortex & 44.83 & 38.99 & 23.92 & 22.13 & $0.025^{\star \star}$ \\
\hline
\end{tabular}

The Mann-Whitney $U$ test was used for all statistical comparisons.

* healthy control > schizophrenia.

${ }^{\star \star}$ schizophrenia > healthy control.

to show abnormalities in network connectivity in schizophrenia patients, especially in the temporal pole (24). In the thalamus, disruption of cortico-thalamic connectivity is associated with the manifestation of various symptoms of schizophrenia $(52,53)$. Furthermore, the precuneus, prefrontal, temporal, and anterior cingulate areas are putative network hubs in the brain network, and aberrant hub organization has appeared in previous analyses of brain networks in schizophrenia $(4,13,48,54)$. We suggest that a reduction in the properties related to regional network segregation in schizophrenia may contribute to the alteration of the global network organization, with the extent of the alteration being more prominent in certain areas.

The nodal centrality indicators, as well as the mean betweenness centrality, showed results inconsistent with previous studies. While we found that the nodal degree value in the frontal and hippocampus areas and the betweenness centrality value in entorhinal cortex were rather increased in the schizophrenia group, previous studies found decreased degree in the frontal hub (55), and decreased values of normalized betweenness centrality in some areas related to the default network (15). These discrepancies in the properties related to centrality may be affected by differences in thresholding of the networks and the heterogeneity of the subject groups.

In the case of continuous network damage, a change in a parameter reflects the overall performance, such as the robustness and stability of the network (18). When evaluating robustness using global efficiency, there was no significant interaction between the number of removed ROIs and the 

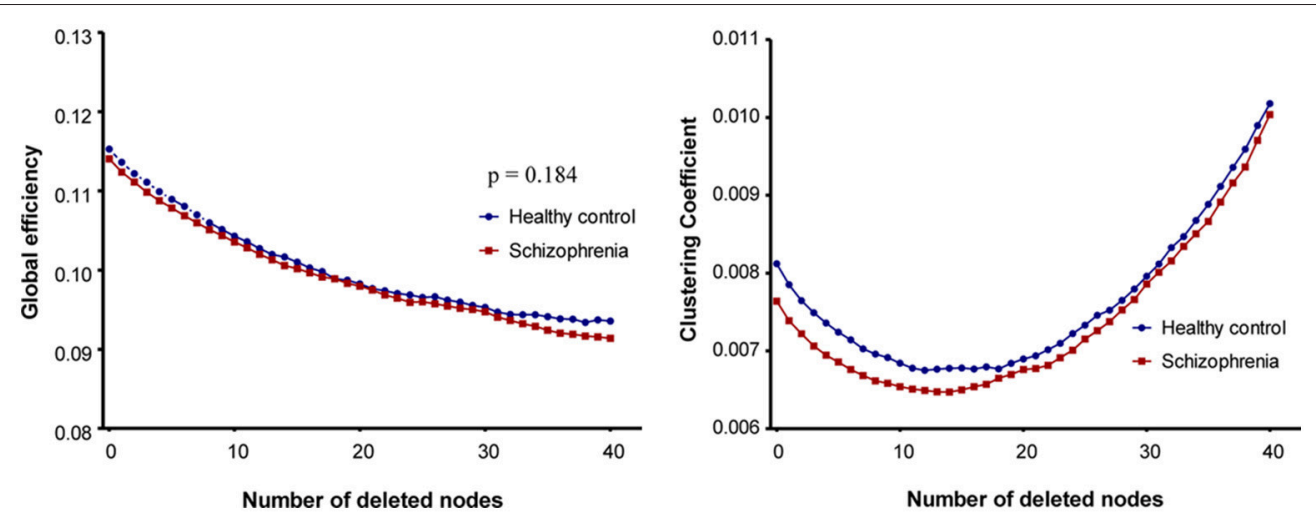

FIGURE 1 | Plots of robustness analysis in schizophrenia patients and healthy control subjects. In case of global efficiency, a linear mixed model was used to assess the group-by-number of removed nodes interaction.

study group. To assess the robustness of anatomical brain networks, a previous study used area-under-the-curve analyses with plots based on the largest cluster size and fraction of node deletions, and detected decreased robustness in schizophrenia (19). Differences in the methods for comparing robustness and image processing might have affected the discrepancies in the results between studies. The clustering coefficient showed a change of $U$ shape when the ROI was repeatedly removed, which is suggestive of a limitation in the use of linear mixed models to compare robustness. This limitation is considered to be due to the reduced number of possible connections between neighboring nodes, which is a denominator in calculating the clustering coefficient.

A significant positive association between CTT-1 t-score and local efficiency and mean connectivity strength was found in the patient group, which is partly consistent with previous studies examining the relationship between processing speed measured by the verbal fluency test and the functional brain network (21). In other words, the degree of network segregation and connectivity strength could be an objective measure of visuomotor speed. Mean connectivity strength was positively correlated with CTT-2 tscore, suggesting that connectivity strength may also be related to executive function and working memory. By contrast, mean betweenness centrality was negatively correlated with CTT1 t-score. IQ raw score in the control group showed a significant correlation with network properties, such as local efficiency, clustering coefficient, and mean connectivity strength, which is consistent with the results of a previous study (47).

However, the network properties showed no significant associations with GAF and PANSS total score or a significant relationship with PANSS positive and negative subscale scores in further analysis. These results are inconsistent with those of previous reports showing associations between symptom severity and reduced levels of overall connectivity and global efficiency $(56,57)$. Further studies, using both anatomical and functional network analysis in combination with a study of the relationship between baseline network characteristics and longitudinal outcome, will be needed to address this issue more clearly.

Although structural connectivity is closely related to functional connectivity $(58,59)$ previous network studies showed inconsistency between these two connectivities of brain network (57). In agreement with our study, many previous studies have reported consistently reduced structural connectivity in schizophrenia patient group (59-61). However, studies of functional connectivity have reported decreased connectivity $(21,62)$, although increased connectivity has also been reported, particularly with respect to hyper-connectivity in the frontal area $(63,64)$. In addition, although no significant differences were found in the present study, robustness to targeted attack on network hubs in schizophrenia was found to be decreased in anatomical network analysis $(19,45)$, and increased in functional network analysis (65). These differences cannot be adequately explained by methodological issues alone. Future research, using DTI and fMRI simultaneously and investigating the difference between structural and functional networks, will be needed to expand understanding of how functional connectivity affects structural connectivity.

There are some limitations to this study which need to be addressed. First, as the diffusion imaging technique relies on water diffusion parameters and its spatial resolution is relatively low compared to the actual size of nerve fibers, diffusion MRI has difficulties with resolving complex fiber organizations, such as crossing, converging, diverging, and kissing fibers $(66,67)$. Nevertheless, diffusion imaging is currently one of only a very small number of tools that can be used for the in vivo evaluation of structural networks in the human brain. In addition, to increase the sensitivity of fiber reconstruction, we used a direct extension of a probabilistic tractography method to reconstruct the networks. The extended probabilistic tractography with a crossing fiber model improved sensitivity for capturing the complexity of neural fiber organization (11). Second, most of the participants in the schizophrenia group had previously been administered antipsychotic medications, and the possibility that the medication served as a confounding factor in the presented results cannot be excluded. However, in some previous studies, 
TABLE 4 | Relationship between network characteristics and clinical assessments.

\begin{tabular}{|c|c|c|c|c|c|}
\hline & & \multicolumn{2}{|c|}{ Schizophrenia } & \multicolumn{2}{|c|}{ Healthy Control } \\
\hline & & $r$ & $P$ & $r$ & $P$ \\
\hline \multirow{6}{*}{$\begin{array}{l}\text { Global } \\
\text { efficiency }\end{array}$} & FSIQ (raw) & -0.09 & 0.569 & 0.33 & 0.138 \\
\hline & FSIQ (adjusted) & 0.11 & 0.457 & -0.22 & 0.327 \\
\hline & $\mathrm{CTT}-\mathrm{t} 1$ & 0.30 & 0.053 & 0.41 & 0.062 \\
\hline & CTT-t 2 & 0.19 & 0.259 & 0.17 & 0.471 \\
\hline & PANSS & -0.08 & 0.598 & & \\
\hline & GAF & -0.15 & 0.431 & & \\
\hline \multirow{6}{*}{$\begin{array}{l}\text { Local } \\
\text { efficiency }\end{array}$} & FSIQ (raw) & 0.16 & 0.286 & 0.51 & $0.016^{\star}$ \\
\hline & FSIQ (adjusted) & 0.20 & 0.174 & -0.10 & 0.642 \\
\hline & $\mathrm{CTT}-\mathrm{t} 1$ & 0.31 & $0.046^{\star}$ & 0.40 & 0.076 \\
\hline & CTT-t 2 & 0.31 & 0.056 & -0.14 & 0.539 \\
\hline & PANSS & 0.05 & 0.741 & & \\
\hline & GAF & -0.14 & 0.468 & & \\
\hline \multirow{6}{*}{$\begin{array}{l}\text { Clustering } \\
\text { coefficient }\end{array}$} & FSIQ (raw) & 0.18 & 0.238 & 0.52 & $0.014^{\star}$ \\
\hline & FSIQ (adjusted) & 0.19 & 0.198 & -0.09 & 0.677 \\
\hline & $\mathrm{CTT}-\mathrm{t} 1$ & 0.30 & 0.056 & 0.39 & 0.081 \\
\hline & CTT-t 2 & 0.31 & 0.062 & -0.15 & 0.509 \\
\hline & PANSS & 0.07 & 0.675 & & \\
\hline & GAF & -0.14 & 0.465 & & \\
\hline \multirow{6}{*}{$\begin{array}{l}\text { Mean } \\
\text { betweenness } \\
\text { centrality }\end{array}$} & FSIQ (raw) & -0.04 & 0.773 & -0.20 & 0.368 \\
\hline & FSIQ (adjusted) & 0.01 & 0.942 & 0.32 & 0.152 \\
\hline & $\mathrm{CTT}-\mathrm{t} 1$ & -0.34 & $0.025^{\star}$ & -0.23 & 0.309 \\
\hline & CTT-t 2 & -0.21 & 0.201 & 0.01 & 0.951 \\
\hline & PANSS & 0.07 & 0.656 & & \\
\hline & GAF & 0.15 & 0.448 & & \\
\hline \multirow{6}{*}{$\begin{array}{l}\text { Mean } \\
\text { connectivity } \\
\text { strength }\end{array}$} & FSIQ (raw) & 0.11 & 0.471 & 0.49 & $0.020^{*}$ \\
\hline & FSIQ (adjusted) & 0.19 & 0.206 & -0.12 & 0.602 \\
\hline & $\mathrm{CTT}-\mathrm{t} 1$ & 0.43 & $0.005^{\star}$ & 0.35 & 0.120 \\
\hline & CTT-t 2 & 0.36 & $0.028^{\star}$ & -0.13 & 0.579 \\
\hline & PANSS & 0.03 & 0.868 & & \\
\hline & GAF & -0.13 & 0.517 & & \\
\hline
\end{tabular}

FSIQ, Full scale intelligence quotient; CTT-t, $t$-score for Color trails test; GAF, Global assessment of functioning; PANSS, Positive and negative syndrome scale ${ }^{*} p<0.05$.

changes in brain connectivity were also observed in medication naive patients (19). Third, no information was available on the level of cognitive function before the onset of disease in the schizophrenia group, and there was a difference in IQ scores between the study groups at recruitment. Therefore, it is unclear whether the differences in network characteristics

\section{REFERENCES}

1. Rössler W, Salize HJ, van Os J, Riecher-Rössler A. Size of burden of schizophrenia and psychotic disorders. Eur Neuropsychopharmacol. (2005) 15:399-409. doi: 10.1016/j.euroneuro.2005.04.009

2. Kraepelin E. Dementia Praecox and Paraphrenia. Huntington, NY: Krieger Publishing Company (1971).

3. Elvevag B, Goldberg TE. Cognitive impairment in schizophrenia is the core of the disorder. Crit Rev Neurobiol. (2000) 14:1-21. doi: 10.1615/CritRevNeurobiol.v14.i1.10 between groups were influenced by the progression of the disease or differences in cognitive functions present before the onset of schizophrenia. In future studies, it would be helpful to use neurocognitive tests such as the Wide Range Achievement Tests, which could estimate the premorbid intellectual function of the patient and allow clearer interpretation of the results. Finally, we used a relatively small sample size, which may have resulted in poor reproducibility. Thus, a larger sample size in followup studies will be required to demonstrate clear and precise differences in nodal network properties between groups, and to determine whether the conclusions of this study can be generalized to other schizophrenia patients.

\section{CONCLUSION}

We compared brain structural networks between schizophrenia patients and healthy controls, using diffusion MRI probabilistic tractography and graph theory. When the topological network properties were compared, measures related to the global network integrity and segregation were significantly lower in the schizophrenia group. This suggests that schizophrenia could induce damage to the entire brain structure and deterioration of inter-regional connectivity, which results in less effective network organization. In addition, considering that group differences in nodal local efficiency were prominent in several regions, schizophrenia may be a disease characterized by network damage over a wide range of brain areas, although the damage disproportionally affects specific brain regions.

\section{AUTHOR CONTRIBUTIONS}

S-HS and JL: Conceptualization; S-HS, WY, and HK: Acquisition of data; S-HS, JL, SJ, and YK: Formal analysis; S-HS, JL, WY, HK, SJ and YK: Investigation; S-HS and JL: Original draft; All authors contributed to and approved the final manuscript.

\section{ACKNOWLEDGMENTS}

This study was supported by the National Research Foundation of Korea (NRF-2012R1A1A1006514 and NRF2017R1D1A1B03032707 to JL).

\section{SUPPLEMENTARY MATERIAL}

The Supplementary Material for this article can be found online at: https://www.frontiersin.org/articles/10.3389/fpsyt. 2018.00272/full\#supplementary-material

4. Rubinov M, Bullmore E. Schizophrenia and abnormal brain network hubs. Dialogues Clin Neurosci. (2013) 15:339-49.

5. Andreasen NC, O'leary DS, Flaum M, Nopoulos P, Watkins GL, Ponto LLB, et al. Hypofrontality in schizophrenia: distributed dysfunctional circuits in neuroleptic-naive patients. Lancet (1997) 349:1730-4. doi: 10.1016/S0140-6736(96) 08258-X

6. Stephan KE, Baldeweg T, Friston KJ. Synaptic plasticity and dysconnection in schizophrenia. Biol Psychiatry (2006) 59:929-39. doi: 10.1016/j.biopsych.2005.10.005 
7. Edwin Thanarajah S, Han CE, Rotarska-Jagiela A, Singer W, Deichmann R, Maurer K, et al. Abnormal connectional fingerprint in schizophrenia: a novel network analysis of diffusion tensor imaging data. Front Psychiatry (2016) 7:114. doi: 10.3389/fpsyt.2016.00114

8. Behrens TE, Woolrich MW, Jenkinson M, Johansen-Berg H, Nunes RG, Clare $\mathrm{S}$, et al. Characterization and propagation of uncertainty in diffusion-weighted MR imaging. Magn Reson Med. (2003) 50:1077-88. doi: 10.1002/mrm.10609

9. Tuch DS, Reese TG, Wiegell MR, Wedeen VJ. Diffusion MRI of complex neural architecture. Neuron (2003) 40:885-95. doi: 10.1016/S0896-6273(03)00758-X

10. Frank LR. Characterization of anisotropy in high angular resolution diffusionweighted MRI. Magn Reson Med. (2002) 47:1083-99. doi: 10.1002/mrm.10156

11. Behrens TE, Berg HJ, Jbabdi S, Rushworth MF, Woolrich MW. Probabilistic diffusion tractography with multiple fibre orientations: what can we gain? Neuroimage (2007) 34:144-55. doi: 10.1016/j.neuroimage.2006.09.018

12. Bullmore E, Sporns O. Complex brain networks: graph theoretical analysis of structural and functional systems. Nat Rev Neurosci. (2009) 10:186-98. doi: $10.1038 / \mathrm{nrn} 2575$

13. Micheloyannis S. Graph-based network analysis in schizophrenia. World J Psychiatry (2012) 2:1-12. doi: 10.5498/wjp.v2.i1.1

14. Iturria-Medina Y, Sotero RC, Canales-Rodriguez EJ, Aleman-Gomez Y, Melie-Garcia L. Studying the human brain anatomical network via diffusion-weighted MRI and Graph Theory. Neuroimage (2008) 40:1064-76. doi: 10.1016/j.neuroimage.2007.10.060

15. Zhang Y, Lin L, Lin CP, Zhou Y, Chou KH, Lo CY, et al. Abnormal topological organization of structural brain networks in schizophrenia. Schizophr Res. (2012) 141:109-18. doi: 10.1016/j.schres.2012.08.021

16. Skudlarski P, Schretlen DJ, Thaker GK, Stevens MC, Keshavan MS, Sweeney JA, et al. Diffusion tensor imaging white matter endophenotypes in patients with schizophrenia or psychotic bipolar disorder and their relatives. Am J Psychiatry (2013) 170:886-98. doi: 10.1176/appi.ajp.2013.12111448

17. van den Heuvel MP, Fornito A. Brain networks in schizophrenia. Neuropsychol Rev. (2014) 24:32-48. doi: 10.1007/s11065-014-9248-7

18. Kaiser M, Martin R, Andras P, Young MP. Simulation of robustness against lesions of cortical networks. Eur J Neurosci. (2007) 25:3185-92. doi: 10.1111/j.1460-9568.2007.05574.x

19. Zhang R, Wei Q, Kang Z, Zalesky A, Li M, Xu Y, et al. Disrupted brain anatomical connectivity in medication-naive patients with first-episode schizophrenia. Brain Struct Funct. (2015) 220:1145-59. doi: 10.1007/s00429-014-0706-Z

20. Arzouan Y, Moses E, Peled A, Levit-Binnun N. Impaired network stability in schizophrenia revealed by TMS perturbations. Schizophr Res. (2014) 152:3224. doi: 10.1016/j.schres.2013.11.017

21. Lynall M-E, Bassett DS, Kerwin R, McKenna PJ, Kitzbichler M, Muller U, et al. Functional connectivity and brain networks in schizophrenia. J Neurosci. (2010) 30:9477-87. doi: 10.1523/JNEUROSCI.0333-10.2010

22. Sporns O. Network attributes for segregation and integration in the human brain. Curr Opin Neurobiol. (2013) 23:162-71. doi: 10.1016/j.conb.2012.11.015

23. van den Heuvel MP, Sporns O. Network hubs in the human brain. Trends Cogn Sci. (2013) 17:683-96. doi: 10.1016/j.tics.2013.09.012

24. van den Heuvel MP, Mandl RC, Stam CJ, Kahn RS, Hulshoff Pol HE. Aberrant frontal and temporal complex network structure in schizophrenia: a graph theoretical analysis. J Neurosci. (2010) 30:15915-26. doi: 10.1523/JNEUROSCI.2874-10.2010

25. Mukherjee P, Chung S, Berman J, Hess C, Henry R. Diffusion tensor MR imaging and fiber tractography: technical considerations. Am J Neuroradiol. (2008) 29:843-52. doi: 10.3174/ajnr.A1052

26. Fedorov A, Beichel R, Kalpathy-Cramer J, Finet J, Fillion-Robin J-C, Pujol S, et al. 3D Slicer as an image computing platform for the quantitative imaging network. Magn Reson Imaging (2012) 30:1323-41. doi: 10.1016/j.mri.2012.05.001

27. Bhushan C, Haldar JP, Joshi AA, Leahy RM. Correcting susceptibilityinduced distortion in diffusion-weighted MRI using constrained nonrigid registration. In: Signal and Information Processing Association Annual Summit and Conference (APSIPA), Asia-Pacific. Asia-Pacific Signal and Information Processing Association Annual Summit and Conference
(2012) Available online at: http://ieeexplore.ieee.org/stamp/stamp.jsp? tp=\&amp;arnumber $=6412009$.

28. Fischl B. FreeSurfer. Neuroimage (2012) 62:774-81. doi: 10.1016/j.neuroimage.2012.01.021

29. Fischl B, Salat DH, Busa E, Albert M, Dieterich M, Haselgrove C, et al. Whole brain segmentation: automated labeling of neuroanatomical structures in the human brain. Neuron (2002) 33:341-55. doi: 10.1016/S0896-6273(02) 00569-X

30. Fischer B, Modersitzki J, editors. FLIRT: a flexible image registration toolbox. In:International Workshop on Biomedical Image Registration (2003) Berlin, Heidelberg: Springer, 261-70.

31. Jenkinson M, Beckmann CF, Behrens TE, Woolrich MW, Smith SM. Fsl. Neuroimage (2012) 62:782-90. doi: 10.1016/j.neuroimage.2011.09.015

32. Rubinov M, Sporns O. Complex network measures of brain connectivity: uses and interpretations. Neuroimage (2010) 52:1059-69. doi: 10.1016/j.neuroimage.2009.10.003

33. Latora V, Marchiori M. Efficient behavior of small-world networks. Phys Rev Lett. (2001) 87:198701. doi: 10.1103/PhysRevLett.87.198701

34. Freeman LC. A set of measures of centrality based on betweenness. Sociometry (1977) 40:35-41. doi: 10.2307/3033543

35. Cao Q, Shu N, An L, Wang P, Sun L, Xia M-R, et al. Probabilistic diffusion tractography and graph theory analysis reveal abnormal white matter structural connectivity networks in drug-naive boys with attention deficit/hyperactivity disorder. J Neurosci. (2013) 33:10676-87. doi: 10.1523/JNEUROSCI.4793-12.2013

36. Watts DJ, Strogatz SH. Collective dynamics of 'small-world'networks. Nature (1998) 393:440.

37. Kay SR, Fiszbein A, Opfer LA. The positive and negative syndrome scale (PANSS) for schizophrenia. Schizophr Bull. (1987) 13:261. doi: 10.1093/schbul/13.2.261

38. Yi JS, Ahn YM, Shin HK, An SK, Joo YH, Kim SH, et al. Reliability and validity of the Korean version of the Positive and negative syndrome scale. J Korean Neuropsychiatr Assoc. (2001) 40:1090-105.

39. Hwang S, Kim J, Park G, Choi J, Hong S. Korean Wechsler Adult Intelligence Scale-(K-WAIS-IV). Daegu: Korea Psychology (2013).

40. D’Elia LF, Satz P, Uchiyama C, White T. Color Trails Test Professional Manual. In: Psychological Assessment Resources. Odessa, FL (1996). doi: 10.1097/00002093-200204000-00003

41. Gonzalez-Blanch C, Alvarez-Jimenez M, Rodriguez-Sanchez JM, PerezIglesias R, Vazquez-Barquero JL, Crespo-Facorro B. Cognitive functioning in the early course of first-episode schizophrenia spectrum disorders: timing and patterns. Eur Arch Psychiatry Clin Neurosci. (2006) 256:364-71. doi: 10.1007/s00406-006-0646-6

42. Yi J, Chung M, Lee B, Lee S. Reliability and validity of the Korean version of global assessment of functioning scale. Korean J Schizophr Res. (2003) 6:118-24.

43. Lee JY, Cho MJ, Kwon JS. Global assessment of functioning scale and social and occupational functioning scale. Korean J Psychopharmacol. (2006) 17:122-7.

44. Benjamini Y, Hochberg Y. Controlling the false discovery rate: a practical and powerful approach to multiple testing. J R Stat Soc Series B (1995) 57:289-300.

45. Griffa A, Baumann PS, Ferrari C, Do KQ, Conus P, Thiran JP, et al. Characterizing the connectome in schizophrenia with diffusion spectrum imaging. Hum Brain Mapp. (2015) 36:354-66. doi: 10.1002/hbm.22633

46. Liu Y, Liang M, Zhou Y, He Y, Hao Y, Song M, et al. Disrupted small-world networks in schizophrenia. Brain (2008) 131:945-61. doi: 10.1093/brain/awn018

47. Zalesky A, Fornito A, Seal ML, Cocchi L, Westin CF, Bullmore ET, et al. Disrupted axonal fiber connectivity in schizophrenia. Biol Psychiatry (2011) 69:80-9. doi: 10.1016/j.biopsych.2010.08.022

48. van den Heuvel MP, Sporns O, Collin G, Scheewe T, Mandl RC, Cahn W, et al. Abnormal rich club organization and functional brain dynamics in schizophrenia. JAMA Psychiatry (2013) 70:783-92. doi: 10.1001/jamapsychiatry.2013.1328

49. Ballmaier M, Schlagenhauf F, Toga AW, Gallinat J, Koslowski M, Zoli $\mathrm{M}$, et al. Regional patterns and clinical correlates of basal ganglia 
morphology in non-medicated schizophrenia. Schizophr Res. (2008) 106:1407. doi: 10.1016/j.schres.2008.08.025

50. Duan M, Chen X, He H, Jiang Y, Jiang S, Xie Q, et al. Altered basal ganglia network integration in schizophrenia. Front Hum Neurosci. (2015) 9:561. doi: 10.3389/fnhum.2015.00561

51. Sarpal DK, Argyelan M, Robinson DG, Szeszko PR, Karlsgodt KH, John M, et al. Baseline striatal functional connectivity as a predictor of response to antipsychotic drug treatment. Am J Psychiatry (2016) 173:69-77. doi: 10.1176/appi.ajp.2015.14121571

52. Anticevic A, Cole MW, Repovs G, Murray JD, Brumbaugh MS, Winkler AM, et al. Characterizing thalamo-cortical disturbances in schizophrenia and bipolar illness. Cerebral Cortex (2014) 24:3116-30. doi: 10.1093/cercor/bht165

53. Kubota M, Miyata J, Sasamoto A, Sugihara G, Yoshida H, Kawada R, et al. Thalamocortical disconnection in the orbitofrontal region associated with cortical thinning in schizophrenia. JAMA Psychiatry (2013) 70:12-21. doi: 10.1001/archgenpsychiatry.2012.1023

54. Fitzsimmons J, Kubicki M, Shenton ME. Review of functional and anatomical brain connectivity findings in schizophrenia. Curr Opin Psychiatry (2013) 26:172-87. doi: 10.1097/YCO.0b013e32835d9e6a

55. Bassett DS, Bullmore E, Verchinski BA, Mattay VS, Weinberger DR, Meyer-Lindenberg A. Hierarchical organization of human cortical networks in health and schizophrenia. J Neurosci. (2008) 28:9239-48. doi: 10.1523/JNEUROSCI.1929-08.2008

56. Wang Q, Su TP, Zhou Y, Chou KH, Chen IY, Jiang T, et al. Anatomical insights into disrupted small-world networks in schizophrenia. Neuroimage (2012) 59:1085-93. doi: 10.1016/j.neuroimage.2011.09.035

57. Skudlarski P, Jagannathan K, Anderson K, Stevens MC, Calhoun VD, Skudlarska BA, et al. Brain connectivity is not only lower but different in schizophrenia: a combined anatomical and functional approach. Biol Psychiatry (2010) 68:61-9. doi: 10.1016/j.biopsych.2010.03.035

58. Rubinov M, Sporns O, van Leeuwen C, Breakspear M. Symbiotic relationship between brain structure and dynamics. BMC Neurosci. (2009) 10:55. doi: 10.1186/1471-2202-10-55

59. Fornito A, Bullmore ET. Reconciling abnormalities of brain network structure and function in schizophrenia. Curr Opin Neurobiol. (2015) 30:44-50. doi: 10.1016/j.conb.2014.08.006

60. Pettersson-Yeo W, Allen P, Benetti S, McGuire P, Mechelli A. Dysconnectivity in schizophrenia: where are we now? Neurosci Biobehav Rev. (2011) 35:1110-24. doi: 10.1016/j.neubiorev.2010.11.004
61. Burns J, Job D, Bastin M, Whalley H, Macgillivray T, Johnstone E, et al. Structural disconnectivity in schizophrenia: a diffusion tensor magnetic resonance imaging study. Br J Psychiatry (2003) 182:439-43. doi: 10.1192/bjp.182.5.439

62. Fornito A, Yoon J, Zalesky A, Bullmore ET, Carter CS. General and specific functional connectivity disturbances in first-episode schizophrenia during cognitive control performance. Biol Psychiatry (2011) 70:64-72. doi: 10.1016/j.biopsych.2011.02.019

63. Anticevic A, Hu X, Xiao Y, Hu J, Li F, Bi F, et al. Early-course unmedicated schizophrenia patients exhibit elevated prefrontal connectivity associated with longitudinal change. J Neurosci. (2015) 35:267-86. doi: 10.1523/JNEUROSCI.2310-14.2015

64. Zhang D, Guo L, Hu X, Li K, Zhao Q, Liu T. Increased cortico-subcortical functional connectivity in schizophrenia. Brain Imaging Behav. (2012) 6:2735. doi: 10.1007/s11682-011-9138-Z

65. Lo CYZ, Su TW, Huang CC, Hung CC, Chen WL, Lan $\mathrm{TH}$, et al. Randomization and resilience of brain functional networks as systems-level endophenotypes of schizophrenia. Proc Natl Acad Sci USA. (2015) 112:9123-8. doi: 10.1073/pnas.1502 052112

66. Jbabdi S, Johansen-Berg H. Tractography: where do we go from here? Brain Connect. (2011) 1:169-83. doi: 10.1089/brain. 2011.0033

67. Bonilha L, Gleichgerrcht E, Fridriksson J, Rorden C, Breedlove JL, Nesland T, et al. Reproducibility of the structural brain connectome derived from diffusion tensor imaging. PLoS ONE (2015) 10:e0135247. doi: 10.1371/journal.pone.0135247

Conflict of Interest Statement: The authors declare that the research was conducted in the absence of any commercial or financial relationships that could be construed as a potential conflict of interest.

Copyright (c) 2018 Shon, Yoon, Kim, Joo, Kim and Lee. This is an open-access article distributed under the terms of the Creative Commons Attribution License (CC $B Y)$. The use, distribution or reproduction in other forums is permitted, provided the original author(s) and the copyright owner are credited and that the original publication in this journal is cited, in accordance with accepted academic practice. No use, distribution or reproduction is permitted which does not comply with these terms. 